Enferm Bras 2019;1895):612-24

https://doi.org/10.33233/eb.v18i5.2478

\title{
ARTIGO ORIGINAL \\ Cuidados paliativos em unidade de atendimento crítico: saberes de uma equipe multiprofissional
}

\author{
Simone Conceição Oliveira Baptista*, Carina Marinho Picanço**
}

${ }^{*}$ Enfermeira especialista em Terapia Intensiva através do Programa de Residência da UNIFACS/SESAB, Metodologia do Ensino Superior, UTI Pediátrica e UTI Neonatal pela Faculdade de Tecnologia e Ciências, Especialização em Dermatologia pela AVM, **Enfermeira mestre em Enfermagem, especialista em Terapia Intensiva pela UFBA, Coordenadora da Comissão de Residência Multiprofissional em Saúde do Hospital Geral Roberto Santos

Recebido 1 de agosto de 2018; aceito 15 de maio de 2019.

Correspondência: Simone Conceição Oliveira Baptista, Conjunto Colinas de Pituaçu, Via B-1, Bloco 643/104 São Marcos 41250-520 Salvador BA

Simone Conceição Oliveira Baptista: baptista.simone@gmail.com

Carina Marinho Picanço: carinampicanco@gmail.com

\section{Resumo}

Introdução: Os cuidados paliativos consistem na assistência promovida por uma equipe multidisciplinar, que objetiva a melhoria da qualidade de vida do paciente e de seus familiares diante de uma doença que ameace a vida, por meio da prevenção e do alívio do sofrimento, da identificação precoce, avaliação impecável e tratamento de dor e demais sintomas físicos, sociais, psicológicos e espirituais. Objetivo: Conhecer os saberes da equipe multiprofissional sobre cuidados paliativos em unidade de atendimento crítico. Metodologia: Pesquisa exploratória descritiva, de natureza qualitativa. Os dados foram analisados segundo o método de análise de conteúdo temático de Bardin e desenvolvido com 65 participantes da equipe multiprofissional de um hospital de caráter privado de médio porte, localizado na área urbana da cidade de Salvador/BA. Resultados: Após tratamento dos resultados, emergiram as seguintes categorias temáticas: Compreendendo a qualidade de vida como essência do cuidado paliativo; Dificuldade de compreensão do melhor momento para início dos cuidados paliativos; Desconhecendo a organização dos cuidados paliativos de acordo com o grau de complexidade da doença e Paliação exclusiva: Quando as medidas heroicas e terapias fúteis provocam dor e sofrimento. Conclusão: A maioria dos profissionais de saúde que participaram desta pesquisa reconhece a necessidade de qualidade de vida e conforto diante do paciente que tem uma doença que ameace a vida. No entanto, desconhecem os conceitos preconizados sobre cuidados paliativos, e algumas premissas, como quando se deve iniciar, as fases que compõe, além da falta de entendimento sobre o significado de cuidados paliativos exclusivos.

Palavras-chave: cuidados paliativos, unidades de terapia intensiva, equipe de assistência ao paciente.

\footnotetext{
Abstract

Palliative care in a critical care unit: knowledge of a multiprofessional team

Introduction: Palliative care consists of the assistance promoted by a multidisciplinary team that aims to improve the quality of life of the patient and his/her relatives in the face of a life-threatening illness, through prevention and relief of suffering, early identification, impeccable evaluation and treatment of pain and other physical, social, psychological and spiritual symptoms. Objective: To know the knowledge of the multiprofessional team about palliative care in a critical care unit. Methodology: Descriptive and qualitative exploratory research. The data were analyzed according to the thematic content analysis method of Bardin and developed with 65 participants of the multiprofessional team of a private hospital of medium size, located in the urban area of ??the city of Salvador/BA. Results: The following thematic categories emerged: Understanding quality of life as the essence of palliative care; Difficulty understanding the best time to start palliative care; Unaware of the organization of palliative care according to the degree of complexity of the
} 
disease and exclusive palliation: When heroic measures and futile therapies cause pain and suffering. Conclusion: Most health professionals who participated in this research recognize the need for quality of life and comfort in front of the patient who has a disease that threatens life. However, they are unaware of the concepts of palliative care advocated, and some premises, such as when to start, the phases that make up, as well as the lack of understanding about the meaning of exclusive palliative care.

Key-words: palliative care, intensive care units, patient care team.

\section{Resumen}

Cuidados paliativos en una unidad de cuidados críticos: conocimiento de un equipo multiprofesional

Introducción: Los cuidados paliativos son la asistencia brindada por un equipo multidisciplinario, cuyo objetivo es mejorar la calidad de vida de los pacientes y sus familias frente a una enfermedad potencialmente mortal, mediante la prevención y el alivio del sufrimiento, la identificación temprana, evaluación y tratamiento impecables del dolor y otros síntomas físicos, sociales, psicológicos y espirituales. Objetivo: Conocer el conocimiento del equipo multiprofesional sobre cuidados paliativos en una unidad de cuidados críticos. Metodología: Investigación exploratoria descriptiva, de naturaleza cualitativa. los datos se analizaron de acuerdo con el método de análisis de contenido temático de Bardin y se desarrollaron con 65 participantes del equipo multiprofesional de un hospital privado de tamaño mediano, ubicado en el área urbana de Salvador/BA. Resultados: Después del tratamiento de los resultados, surgieron las siguientes categorías temáticas: entender la calidad de vida como la esencia de los cuidados paliativos; Dificultad para entender el mejor momento para comenzar los cuidados paliativos; Sin darse cuenta de la organización de los cuidados paliativos de acuerdo con el grado de complejidad de la enfermedad y la paliación exclusiva: cuando las medidas heroicas y las terapias inútiles causan dolor y sufrimiento. Conclusión: La mayoría de los profesionales de la salud que participaron en esta investigación reconocen la necesidad de calidad de vida y comodidad frente al paciente que tiene una enfermedad potencialmente mortal. Sin embargo, desconocen los conceptos recomendados de cuidados paliativos y algunas suposiciones, como cuándo comenzar, las fases que conforman, además de la falta de comprensión sobre el significado de los cuidados paliativos exclusivos.

Palabras-clave: cuidados paliativos, unidades de cuidados intensivos, equipo de atención al paciente.

\section{Introdução}

Os cuidados paliativos (CP) consistem na assistência promovida por uma equipe multidisciplinar, que objetiva a melhoria da qualidade de vida do paciente e de seus familiares diante de uma doença que ameace a vida, por meio da prevenção e do alívio do sofrimento, da identificação precoce, avaliação impecável e tratamento de dor e demais sintomas físicos, sociais, psicológicos e espirituais [1].

Os CP nasceram, primordialmente, para atender aos pacientes oncológicos em estágio avançado da doença, contudo, hoje se estendem a todos àqueles que tenham alguma doença que ameacem a vida, que cause dor intensa, sintomas físicos, emocionais e/ou espirituais, tornando a vida extremamente intolerável. Cuidados direcionados a pessoa considerada pela ciência sem possibilidade da cura ${ }^{1}$, mas que podem ser cuidadas visando assegurar conforto e dignidade no processo de morrer e na morte [2].

Hoje em dia, os cuidados paliativos começam a ser compreendidos como a fase de cuidados que antecede a morte, e torna-se uma prática multidisciplinar que envolve interações com outras partes relevantes dos sistemas de cuidados de saúde. Os cuidados paliativos tornaram-se uma disciplina prática com vistas a melhorar os cuidados daqueles que estejam no final da vida [3]. ${ }^{1}$

\footnotetext{
${ }^{1} \mathrm{O}$ termo "doença ameaçadora à vida" é mais apropriado para designar o paciente em risco iminente de morte [13]. Em respeito aos autores será mantido o termo.
} 
Ademais, o crescimento tecnológico de artifícios que facilitam à prolongação da vida e a cura das enfermidades pode ser presenciado e constatado nos hospitais, sobretudo nas Unidades de Terapia Intensiva (UTIs) na contemporaneidade. Neste sentido, as tecnologias altamente sofisticadas encontram-se a disposição da equipe de saúde, que atua buscando a cura e o controle das doenças dos pacientes [4]. Esta mesma tecnologia que muitas vezes e em certas circunstâncias pode salvar vidas, em outras, torna o final da vida extremamente doloroso, sofrido e solitário.

Outro aspecto que representa um enorme desafio para os profissionais da saúde, diz respeito à dificuldade de diálogo com os pacientes e familiares sobre o processo de doença, sobretudo em situações críticas, internações em unidade de terapia intensiva (UTI) e processo de morte-morrer. Contudo, é muito importante que essa comunicação seja clara, livre de ruídos e seja com o paciente e familiar [5].

Assim, para implementação adequada dos cuidados paliativos pelos profissionais da saúde, melhora a atenção prestada às pessoas que estão no final de suas vidas, e melhora os cuidados e a assistência às suas famílias e amigos, melhora a qualidade de vida, melhora o controle dos sintomas e até reduz o uso de recursos da saúde [6].

Onde quer que sejam postos em prática, os cuidados paliativos, fundamenta-se em valores e princípios como a dignidade do paciente e familiar, aos cuidados que apresentem compaixão para com o paciente e a família, equidade no acesso aos serviços de cuidados paliativos, respeito pelo paciente, pela família e por quem cuidam do mesmo, lutar por conseguir atender os desejos do paciente, da família e da comunidade, prosseguir na excelência da provisão dos cuidados e de apoio e responsabilidade para com os pacientes, as famílias e comunidade em geral [7].

Muito embora os cuidados críticos e os cuidados paliativos possam parecer estar em lados opostos do espectro, uma inspeção mais próxima revela muitas semelhanças. Historicamente, as duas especialidades desenvolvem-se em datas próximas, e ambas cuidam de pacientes e suas famílias nos momentos de extrema vulnerabilidade e necessidade [8].

Ademais, a morte ainda incomoda e desafia a onipotência dos profissionais de saúde, ensinados apenas a cuidar da vida, mas não da morte. Lidar com a morte é angustiante e desgastante, gera sentimentos de impotência, frustração e insegurança, pois não estão preparados para lidar com todos os sentimentos negativos e ambivalentes presentes na situação. Neste sentido podemos pensar que combater a morte pode dar falsa ideia de força e controle, fato que nos obriga a repensar valores e considerar uma noção mais abrangente do cuidado paliativo, levando em conta a qualidade de sua morte [9].

A escolha deste tema expressa primeiramente os anseios pessoais de conhecer mais detalhadamente o papel da equipe multiprofissional diante do paciente em cuidados paliativos da qual a pesquisadora teve contato durante a sua experiência profissional na residência multiprofissional em saúde com ênfase em terapia intensiva. Neste cenário observaram-se divergências de condutas no lidar com paciente em CP, além da dificuldade velada dos profissionais em lidar com o processo de morte e morrer e em reconhecer a necessidade da abordagem aos pacientes elegíveis. Entende-se atualmente que durante a evolução da doença os cuidados paliativos e curativos andam lado a lado, não sendo necessariamente excludentes.

Nos períodos de exacerbação ou descompensação da doença, ao mesmo tempo em que ocorre a intensificação do cuidado curativo, deve haver também a intensificação do cuidado paliativo, buscando o controle adequado dos sintomas. Ainda era perceptível uma assistência multiprofissional prestada ao paciente diante de uma doença ameaçadora a vida no modelo "tudo ou nada" onde o cuidado paliativo não estava integrado ao cuidado curativo.

Neste sentido, esta pesquisa torna-se relevante por possibilitar reflexões por parte da equipe de profissionais que atuam em UTI, sobre sua prática assistida ao paciente com doença ameaçadora à vida que necessitem de CP. Repensar um cuidado sensível, humanístico e holístico, elencado no respeito e na participação e emancipação do doente e no apoio aos familiares no processo de luto.

Diante do exposto, questiona-se: Quais são os saberes da equipe multiprofissional sobre cuidados paliativos em unidade de atendimento crítico? Para tanto, esta pesquisa tem como objetivo principal conhecer os saberes da equipe multiprofissional sobre cuidados paliativos em unidade de atendimento crítico. 
"Consiste em cuidados que expressem qualidade da assistência prestada pela equipe multiprofissional visando o conforto ao paciente e da família e qualidade de vida diante de uma patologia que ameaça a vida” (E1).

A metodologia utilizada para a elaboração desse estudo foi uma pesquisa exploratória descritiva, de natureza qualitativa.

Analisar significa estudar, decompor, dissecar, dividir, interpretar. A análise de um texto refere-se ao processo de conhecimento de determinada realidade e implica o exame sistemático dos elementos; portanto, é decompor um todo em suas partes, a fim de poder efetuar um estudo mais completo, encontrando o elemento-chave do autor, determinar as relações que prevalecem nas partes constituídas, compreendendo a maneira pela qual estão organizadas, e estruturar as ideias de maneira hierárquica [10].

É a análise que vai permitir observar componentes de um conjunto, perceber suas possíveis relações, ou seja, passar de uma ideia chave para um conjunto de ideias mais especificas, passar a generalização e, finalmente, à crítica [10].

Este estudo foi desenvolvido através da coleta de dados tendo como instrumento um formulário que foi preenchido pelo participante tendo por objetivo descrever completamente um fenômeno.

\section{Local de estudo}

O local de estudo foi realizado em um hospital de caráter privado de médio porte, localizado na área urbana da cidade de Salvador/BA, inaugurado em 2000. Sendo referência em emergência adulto, pediátrica, ortopédica e realiza cirurgias de pequena, média e alta complexidade bem como cardíacas. A instituição possui um andar, divididos em área administrativa, ambulatórios, centros especializados, centro cirúrgico, unidades de clínica médica e cirúrgica.

Atende as especialidades de neurologia, cardiologia, dermatologia, ginecologia, reumatologia, urologia, gastropediatria, ortopedia e traumatologia, proctologia, coloproctologia, mastologia, oftalmologia e pediatria. Conta com os serviços de apoio (almoxarifado, farmácia, copa, refeitório, laboratório) e diagnóstico (raios-X, ultrassonografia, tomografia computadorizada, endoscopia digestiva, exames oftalmológicos, ginecológicos, eletrocardiograma, ecocardiograma, mapa, Holter, teste ergométrico e urofluxometria). Possuem 51 leitos unidade de internação, 08 leitos na unidade de emergência e 20 leitos de UTI Geral para adultos, totalizando 89 leitos. O cenário de escolha para este estudo foi a UTI Geral Adulto.

A UTI Geral Adulto possui um perfil de pacientes bastante diversificados, pacientes em pós-operatório, com urgências em geral, inclusive dialíticas. Especialidades de neurologia, nefrologia, infectologia, cirurgia cardíaca e cirurgia geral.

\section{Participantes do estudo}

Foram convidados para participar desta pesquisa, médicos, enfermeiros, fisioterapeutas, nutricionistas, fonoaudióloga, psicólogos e técnicos de enfermagem tendo como critério de inclusão a atuação de no mínimo dois anos em (UTI), por considerar que nesse período os profissionais podem ter desenvolvido uma concepção mais consolidada sobre a temática. Tendo como critério de exclusão àqueles que não aceitarem participar da pesquisa, estavam afastados do serviço e não ter tempo de atuação mínima dois anos em UTI.

A UTI selecionada tinha um total de 17 médicos, 19 enfermeiros, 32 técnicos de enfermagem, 13 fisioterapeutas, 5 nutricionistas, 3 psicólogos e 1 fonoaudióloga.

Do total de médicos, 8 participaram do estudo, 6 recusaram a participar e 03 não atenderam o critério de inclusão. Do total de enfermeiros, 16 foram incluídos no estudo, 1 recusou a participação, 1 desistiu e 1 não atendeu ao critério de inclusão. Entre os 32 técnicos de enfermagem, 25 aceitaram participar do estudo, além de atender o critério de inclusão, 3 recusaram a participar do estudo, 1 não atendia ao critério de inclusão e 03 estavam afastados pela Previdência Social.

Dos 13 fisioterapeutas, 1 não atendeu o critério de inclusão. Entre os 3 psicólogos, 2 desistiram de participar da pesquisa. Dos 5 nutricionistas, 2 atenderam ao critério de inclusão, 2 
recusaram e 1 desistiu durante o processo. E uma fonoaudióloga. Do total de 90 profissionais do local de estudo, obteve-se um quantitativo de 65 participantes.

Para realizar o preenchimento do formulário os encontros foram previamente agendados e realizados fora do horário de trabalho do profissional em ambiente reservado. Foi utilizada uma amostragem aleatória para a escolha dos participantes do estudo.

\section{Aspectos éticos}

Respeitando os preceitos éticos da pesquisa, foi encaminhado um ofício à Direção e ao Núcleo de Pesquisa e Ensino do referido hospital, solicitando autorização para a realização desse estudo, após obter o parecer favorável do Comitê de Ética do Hospital Geral Professor Roberto Santos. Antes de iniciar a abordagem aos entrevistados, foi apresentado o projeto para os coordenadores dos respectivos setores definidos.

Os profissionais foram abordados fora do seu horário de trabalho, onde foi explicada a intencionalidade e, apresentado o parecer favorável do Comitê de Ética, bem como, foram fornecidas informações sobre o estudo, seu objetivo e o instrumento que seria utilizado para a coleta dos dados. Os participantes ficaram à vontade para a escolha em participar ou não da pesquisa, assim como, o melhor momento para tal. Quando os profissionais se sentiram à vontade e disponíveis, após o término da jornada de trabalho, elas comunicavam que estavam prontos. Assim, foi definido o melhor local para a coleta, nas respectivas salas de confortos de enfermagem, de médicos e de técnicos de enfermagem da UTI, por considerar serem locais reservados e capazes de proporcionar privacidade para os entrevistados.

Esta pesquisa atendeu o que foi estabelecido nas Diretrizes do Conselho Nacional de Saúde e na Resolução o 466 de 12 de dezembro de 2012 sobre pesquisas envolvendo seres humanos e a aprovado, pelo Comitê de Ética em Pesquisa (CEP) sob o CAEE número: 79251217.7.0000.5028 e pela administração da instituição de estudo.

Após o fornecimento de informações sobre o trabalho, inclusive seu objetivo e justificativa para o estudo, além de explicitar o instrumento que foi utilizado, foi entregue a cada membro da pesquisa o Termo de Consentimento Informado Livre e Esclarecido (TCLE) em duas vias devidamente assinadas.

Neste foi garantido a preservando o anonimato, deixando claro sobre a livre escolha, sem uso de indução, coação ou incentivo financeiro, podendo o participante colaborar e/ou desistir em qualquer fase da pesquisa.

Os formulários foram lidos, transcritos e analisados logo após a realização das entrevistas. Mantida a integridade e fidedignidade dos relatos, sendo analisados de forma a não modificar o conteúdo das escritas. Após o término da pesquisa, os formulários já estão arquivados confidencialmente e permanecerão por um intervalo de tempo de cinco anos e serão incinerados após este período.

A fim de manter o anonimato dos depoentes, foram identificados por códigos seguidos de numeração. Assim denominadas: médicos ( $M 1, M 2, M 3 \ldots)$, enfermeiros ( $E 1$, E2, E3...), fisioterapeutas ( $F 1, F 2, F 3 \ldots)$, nutricionistas (N1, N2, N3...), psicólogos (P1, P2, P3...), fonoaudióloga (A1) e técnicos de enfermagem (T1, T2, T3...).

\section{Técnicas/ instrumento de coleta de dados}

A coleta de dados foi realizada através do preenchimento de um formulário. O roteiro do formulário, previamente elaborado, testado e corrigido, constou de questões que caracterizavam o perfil sócio-demográfico dos participantes como idade, sexo, tempo de experiência e formação, cursos de pós-graduação, e questões específicas e objetivas sobre o tema.

$\mathrm{O}$ instrumento de coleta de dados foi composto por duas partes. A primeira continha questões fechadas dados de caracterização sócio-demográfica e de formação profissional dos participantes do estudo e, a segunda parte foi composta por questões norteadoras a exemplo: 1) Qual o conceito de cuidados paliativos? 2) Quando se inicia os cuidados paliativos no paciente crítico? 3) Quais fases fazem parte dos cuidados paliativos? 4) O que significa "cuidados paliativos exclusivos"?

Análises dos dados 
Para a abordagem qualitativa, os dados foram analisados segundo o método de análise de conteúdo temático de Bardin [11], que propõe um conjunto de técnicas de análise de comunicação verbal aplicado aos discursos, para obtenção de indicadores, qualitativos ou não, que permitam a descrição do conteúdo das mensagens dos entrevistados. Esse método sistemático e prático é composto por três etapas distintas: a) pré-análise, b) exploração do material, c) tratamento dos resultados a partir das inferências e interpretações.

De acordo com os princípios deste método, as estruturas e elementos do conteúdo foram desmontadas e analisadas por meio do estudo minucioso das palavras e frases que 0 compuseram, procurando seu sentido e intenções, reconhecendo, comparando, avaliando e selecionando-o para esclarecer suas diferentes características e extrair sua significação, utilizando-se o critério temático.

A pré-análise consistiu, inicialmente, da leitura flutuante de cada resposta das entrevistas permitindo ao pesquisador se deixar invadir sobre impressões a respeito do material coletado. Ulteriormente, elas foram lidas exaustivamente e minuciosamente, visando à compreensão profunda do material. Durante a leitura foram destacadas as falas sendo denominadas de núcleos de sentido (unidade de significados). A categorização decorreu na análise e semelhança apresentadas pelas unidades de significação possibilitando definir quatro categorias temáticas. Os resultados foram trabalhados para se tornarem reveladores de significados. Realizado assim, inferências e interpretações baseada na literatura que fundamentaram o estudo, retornando à mesma para contribuir com o processo de reflexão [11].

\section{Resultados e discussão}

"É o conjunto de cuidados ao paciente em fase avançada da doença, que priorizam alívio do sofrimento do mesmo e de sua família" (M9).

\section{Caracterização dos participantes da pesquisa}

Dos 65 participantes do estudo, cinquenta e dois eram mulheres e treze homens, com idade entre 23 e 59 anos. O tempo de formação variou entre três e trinta e seis anos, sendo que destes, o tempo de experiência em UTI, foi de dois a vinte dois anos.

Entre os participantes médicos, enfermeiros, fisioterapeutas, nutricionistas, psicólogos e fonoaudióloga, 1 médico possui doutorado e 1 fisioterapeuta mestrado. E especialização em diversas áreas totaliza 26 membros e no formato de residência 19 profissionais. Dentre os técnicos de enfermagem, onze tinham especialização de nível médio em UTI.

\section{Categorias temáticas}

Partindo-se das ideias centrais dos cuidados paliativos em unidade de atendimento crítico e os saberes de uma equipe multiprofissional, após tratamento dos resultados, emergiram as seguintes categorias temáticas: Compreendendo a qualidade de vida como essência do cuidado paliativo; Dificuldade de compreensão do melhor momento para início dos cuidados paliativos; Desconhecendo a organização dos cuidados paliativos de acordo com o grau de complexidade da doença e Paliação exclusiva: quando as medidas heroicas e terapias fúteis provocam dor e sofrimento.

\section{Categoria 1 - Compreendendo a qualidade de vida como essência do cuidado paliativo}

Embora os profissionais não apontem o conceito total de cuidados paliativos, quando questionados a maioria dos participantes traz em seus discursos um elemento essencial para a abordagem, a necessidade de promover a qualidade de vida, conforme trechos a seguir:

"É fornecer o cuidado ao paciente independente de proposta curativa, buscando bem-estar à qualidade de vida do paciente". (M5)

"Consiste no oferecimento de conforto a família e ao paciente que se encontra na fase terminal da doença, objetivando a melhoria na qualidade de vida no paciente neste período". (F9) 
Efetivamente $\mathrm{CP}$ é uma forma de abordagem que visa à melhoria da qualidade de vida de pacientes e seus familiares que enfrentam doenças ameaçadoras à vida, através da prevenção, da identificação e do tratamento precoce dos sintomas físico, psíquico, espiritual e social e que esses cuidados devem ser prestados a todos os pacientes, em concomitância com os cuidados curativos, sendo a intensidade individualizada de acordo com as necessidades, com os desejos dos pacientes e de seus familiares e com a evolução própria de cada doença [12].

Desta forma, o modo como cada paciente vive a sua terminalidade tem a ver com as circunstâncias em que está sendo cuidado, na dinâmica da ética das relações que se criam entre ele e os profissionais que o assistem. Portanto o enfoque terapêutico é voltado para a qualidade de vida, o controle dos sintomas do doente e o alívio do sofrimento humano apoiado no caráter trans, multi e interdisciplinar dos cuidados paliativos. A terapêutica paliativa é voltada ao controle sintomático e preservação da qualidade de vida para o paciente, sem função curativa, de prolongamento ou de abreviação da sobrevida [13].

Quando a OMS traz em seu conceito que "Cuidados paliativos é uma abordagem que aprimora a qualidade de vida..." pode-se pensar que esta filosofia pode especificar alguns princípios fundamentais, tais como: valorizar a obtenção e a manutenção de um nível ótimo de dor e a administração dos sintomas; os CP afirmam a vida e encaram a morrer como um processo normal; não apressam nem adiam a morte; integram aspectos psicológicos e espirituais dos cuidados do paciente; oferecem sistema de apoio para ajudar os pacientes a viver tão ativamente quanto possível, até o momento da sua morte; ajudam a família no enfretamento da doença do paciente e do luto; a família é uma unidade de cuidados, juntamente com o paciente; os CP exigem uma abordagem inter e multidisciplinar; destinam-se a aprimorar a qualidade de vida e são aplicáveis no estágio inicial da doença ameaçadora da vida, concomitantemente com as modificações da doença e terapias que prolongam a vida.

A assistência aos familiares é um dos aspectos importantes do cuidado global dos pacientes internados na UTI, sendo um dos pilares do cuidado humanizado. Do ponto de vista ético, existe um grande impacto nas relações humanas e profissionais, onde se deve considerar o paciente e a família como uma unidade de cuidados.

A propósito, o cuidado prestado à família ainda merece respeito necessário, tanto no âmbito assistencial quanto no processo formativo dos profissionais. Uma abundante literatura contemporânea evidencia que estratégias assistências voltadas para os familiares (englobando não somente parentes consanguíneos e cônjuges, mas também todos os que fazem parte do círculo afetivo do paciente), como a melhoria de comunicação, prevenção de conflitos e valores e escolhas, e conforto espiritual resultam em maior satisfação e percepção da qualidade de vida e de assistência ao paciente na UTI [14].

Outrossim, ainda nos chamam a atenção para a questão do conforto neste processo, conforme excertos abaixo:

"Consiste em cuidados que expressem qualidade da assistência prestada pela equipe multiprofissional visando o conforto ao paciente e da família e qualidade de vida diante de uma patologia que ameaça a vida". (E1)

"São aqueles cuidados dados aos pacientes ao qual se utilizou de todos os recursos para a manutenção da vida do paciente, seja medicamentoso e com isso se inicia uma medida de conforto". (T2)

"Ações realizadas pela equipe multiprofissional com objetivo de garantir 0 conforto, evitando qualquer medida fútil". (P1)

"É quando o foco do tratamento do paciente deixa de ser curativo para priorizar o conforto do mesmo". (N2)

Para que haja excelência de assistência prestada aos pacientes com doença ameaçadora a vida e os cuidados ao final da vida, é indispensável que a equipe de saúde encontre estratégias para o controle de sintomas físicos, mas que, também, valorize a necessidade de alívio de sofrimentos psicológicos e espirituais presentes nesta situação.

Some-se a isto o controle dos sintomas e melhora da qualidade de vida do paciente em cuidados paliativos exclusivos ser promovido por medidas farmacológicas e não farmacológicas. 
A equipe deve atuar coordenadamente na aplicação de medidas não farmacológicas como horário de visitas mais flexível, musicoterapia, quarto privativo a suspensão de restrições físicas e controle de fatores ambientais, tais como, excesso de barulho. Essas medidas contribuem para a prevenção e controle de delírio em pacientes críticos.

Nos depoimentos, observou-se a preocupação com alívio dos sintomas físicos proporcionando o conforto e diminuição do sofrimento psicossocial. Contudo, nenhum participante relatou a dimensão espiritual. Constatou-se, ainda a inclusão da família como parte do cuidado e as necessidades de garantir qualidade de vida, concordando com a filosofia de cuidados paliativos, definida pela OMS.

Dessa forma, o cuidado paliativo propõe, ao profissional de saúde, o desafio de cuidar com competência científica sem, no entanto, esquecer-se da valorização do ser humano. Para que essas necessidades sejam atendidas, e o cuidado seja integral, é primordial que a equipe de saúde resgate a relação interpessoal empática, sendo fundamental ouvir e torna-se sensível às necessidades dos pacientes, mais do que habilidades técnicas para diagnosticar e tratar. Estes pacientes esperam que a relação com os profissionais de saúde seja alicerçada por compaixão, respeito e empatia, de modo a auxiliá-los no processo de morte, valorizando a sua experiência [15].

Para o cuidado paliativo é necessário que o trabalho multiprofissional procure resgatar os valores éticos e humanos, assim como a autonomia individual. O cuidado deve ser compartilhado, e o paciente no processo de terminalidade merece do profissional toda a benevolência e respeito. Auxiliá-lo em todas as fases deste processo, implica orientá-lo sem coagir, mostrando-lhe os benefícios e desvantagens de cada tratamento, de forma inteligível a seu nível de compreensão [15].

Assim, para que haja qualidade de vida ao paciente portador de doença crônicodegenerativa ameaçadora a vida, o profissional de saúde precisa ter uma atitude humanizada no processo do cuidar. Entende-se que a essência humana está no cuidado, sendo o suporte para inteligência, criatividade e liberdade sendo transposto pelos princípios morais, éticos, valores e atitudes presentes no agir e viver.

A propósito, para a construção do processo saudável é fundamental um cuidado que estimule e permita o indivíduo a tomar consciência do mundo e de si mesmo, sustentando a responsabilidade pela sua transformação e consequentemente melhorar a qualidade de vida. $\mathrm{E}$ para a equipe de saúde é um grande desafio cuidar do ser humano em sua totalidade e integralidade, e ter uma ação efetiva preferencialmente na dor e sofrimento, nas dimensões físicas, psíquica, social e espiritual com competência técnico-científica e humana.

Categoria 2 - Dificuldade de compreensão do melhor momento para início dos cuidados paliativos

Nesta categoria, foi evidenciado que poucos participantes demonstraram 0 conhecimento do momento de encetar cuidados paliativos, de acordo com as falas a seguir: "Sempre, desde a admissão do paciente na UTI". (M5)

"Na admissão". (M8)

"Os cuidados paliativos no paciente crítico devem ser iniciados na sua admissão. Eles podem ser gerais, visando o conforto de todo o paciente admitido com criticidade, minimizando a dor, proporcionando conforto de modo geral, higiene, apoio familiar e psicológico com perspectiva curativa e prognóstico satisfatório". (E8)

"Se iniciam no paciente crítico no momento em que sua internação se inicia". (E9)

"Quando adentra a unidade". (F8)

Em adição, a maioria dos participantes não concebem qual o momento apropriado para iniciar a paliação, associando que esta conduta deverá ser implementada quando o paciente obtiver prognóstico reservado ou em processo de terminalidade da vida: 
"Quando esgotamos o tratamento clínico visando à cura - doença irreversível". (E3)

"Quando a doença é irreversível". (E7)

"A partir da constatação de uma doença irreversível, bem como do momento de identificação do paciente que se encaixa em curta perspectiva de sobrevida". (M7)

"Depois que todas as alternativas de recuperação de saúde (vida) não surtem efeito. Quando não se tem mais prognóstico". (T3)

"Se inicia quando o paciente não tem um prognóstico bom, os cuidados paliativos priorizam a qualidade de vida do paciente mesmo sabendo que $o$ final será a morte". (T7)

"Quando o paciente não responde mais a terapia utilizada; sem prognóstico favorável”. (F5)

"Quando observa-se que não há mais tratamento para a doença do paciente; que o paciente não responde mais ao que já foi feito". (N2)

Além disso, o CP é uma abordagem que deve começar no momento do diagnóstico da doença grave e ameaçadora à vida segundo Organização Mundial de Saúde. Seu objetivo principal é promover a qualidade de vida e aliviar os sintomas de sofrimento durante a doença, visando não só o paciente, mas também seus familiares e às pessoas importantes de sua convivência [16].

Para a American Thoracic Society todos os pacientes de UTI deveriam receber cuidados paliativos, uma vez que todos apresentam doenças que ameaçam a continuidade da vida, e isso não exclui a busca da cura e o cuidado curativo [17].

Entende-se atualmente que durante a evolução da doença os cuidados curativos e paliativos andam lado a lado, não necessariamente excludentes, ao mesmo tempo em que ocorre a intensificação do cuidado curativo, deve haver a intensificação do cuidado paliativo, buscando o controle adequado dos sintomas. Integração entre cuidados curativos e paliativos é essencial, especialmente na UTI, evitando-se a abordagem do "tudo ou nada", conforme a recomendação de diversas sociedades médicas [18].

Neste sentido, é importante entender que os cuidados paliativos são concebidos com estratégia para atender às necessidades de pacientes com doenças graves e de alta complexidade e dos familiares paralelamente ao cuidado curativo no momento da admissão do doente na UTI. Desta forma, todos se beneficiam igualmente, recebendo cuidados de forma integral e digna, aliviando o sofrimento, bem como proporcionando a melhor qualidade de vida possível.

Além disso, acompanhar um ser humano que está sofrendo ou morrendo é um dos maiores desafios que a equipe multiprofissional pode enfrentar. Trata-se de um processo permeado por crises de medo e insegurança. Não há uma maneira correta de acompanhar esses pacientes, porque o morrer de cada um é tão singular quanto o seu viver. Daí a necessidade de se conhecer e implementar adequadamente as fases que compõe os cuidados paliativos, uma vez que será garantido a atenção integral para todos os envolvidos.

Categoria 3 - Desconhecendo a organização dos cuidados paliativos de acordo com o grau de complexidade da doença.

A Câmara Técnica de Terminalidade e Cuidados Paliativos da Associação de Medicina Intensiva Brasileira (AMIB), levando em consideração os conceitos previamente apontados e, tendo em vista a necessidade de que todos os pacientes criticamente enfermos devam receber cuidados paliativos, foi realizado o IIo Fórum do Grupo de Estudos do Fim da Vida do Cone Sul em 2010, na cidade de Brasília. O objetivo deste fórum foi a elaboração de recomendações pertinentes aos cuidados paliativos a serem prestados aos pacientes críticos. 
Como resultado os participantes, baseados em dados de literatura, sugeriram definições, recomendações e ações integradas a serem seguidas para a implantação dos cuidados paliativos propostos aos pacientes criticamente enfermos. Foi também proposto um fluxograma para a prestação de cuidados paliativos na UTI.

O paciente com uma doença ameaçadora de vida pode ser classificado em 04 fases distintas dos cuidados paliativos. A primeira fase é aplicável na maior parte dos pacientes da UTI, quando a recuperação é o desfecho mais provável e os cuidados que buscam a cura e/ou controle da doença é a prioridade. Nesta fase, os CP promovem o adequado controle de sintomas e a comunicação empática com pacientes e familiares. Já na fase 02 a recuperação ou o controle são improváveis, pode-se, por meio do consenso com paciente e/ou família, priorizar os cuidados que buscam o que o paciente entende por conforto e qualidade de vida, utilizando intervenções para controle da doença de maneira proporcional a este objetivo prioritário do cuidado. Durante a terceira fase acontece em situações em que a morte apresenta-se iminente ou inevitável, o CP pode ser exclusivo [13].

Em outro estudo complementando esta recomendação a quarta e última fase caracterizase também pelo suporte e apoio aos familiares diante do óbito do paciente [15].

Contrariamente ao que foi exposto, os participantes deixam evidentes, que desconhecem quais as fases compõem os cuidados paliativos.

"Fases não sei, não consigo definir". (A1)

"Desconheço essa classificação por fases". (E14)

"Em todas as fases: controle dos sintomas (dor, desconforto); Fase I: facilitar presença dos familiares; respeitar as crenças; proporcionar apoio psicológico. Fase II: possibilitar alta da UTI; Fase III: retirar terapias fúteis". (P1)

"Não tenho conhecimento das fases". (F2)

"Não há fases". (M8)

"Não conheço essa classificação, não faz parte da minha vivência prática tal classificação". (E2)

O déficit de conhecimento da equipe multiprofissional sobre as fases que compõe o $\mathrm{CP}$ pode comprometer a assistência de saúde ao paciente crítico. Cada etapa imprime a necessidade de atenção e medidas específicas para melhora do quadro clínico e definição dos cuidados paliativos exclusivos.

Diante dos discursos, os membros desta equipe demonstraram dificuldades em determinar em qual fase do $\mathrm{CP}$ o paciente se encontra devido à carência de formação teórica e prática para lidar com este momento do processo de terminalidade.

Desta maneira, deduz-se que é de fundamental importância ampliar a discussão e a formação profissional acerca dos cuidados paliativos, aprimorando o currículo através de atividades extensionistas que tratem da morte e dos cuidados a pessoas que está morrendo, com o propósito de conscientização e sensibilização dos profissionais da equipe multiprofissional intensivista para melhor atuarem e discutirem sobre a temática.

Categoria 4 - Paliação exclusiva: quando as medidas heroicas e terapias fúteis provocam dor e sofrimento.

O CP se apoia na visão da ortotanásia que se caracteriza pela morte em seu tempo natural, garantindo a dignidade do indivíduo e promovendo a seu bem-estar, com a finalidade de produzir uma "boa morte". Por conseguinte, o investimento extenuante em esforços da equipe multiprofissional para salvar vidas e todo o aparato tecnológico envolvido no cuidado no ambiente de terapia intensiva é um paradoxo à abordagem paliativa, uma vez que esta traz a proposta de promover conforto ao enfermo, não necessitando de grandes recursos tecnológicos. [8]

Os participantes trazem a importância de se evitar medidas heroicas e terapias fúteis, ou seja, haverá um momento em que não se convém um tratamento curativo: 
"Quando não utiliza métodos invasivos, medidas heroicas priorizando sedação e conforto". (E6)

"Evitar medidas invasivas, priorizar conforto focando em controle de sintomas e evitando medidas fúteis". (M7)

Observa-se também nos discursos uma preocupação em afirmar que a abordagem não tenta promover a cura, de acordo com alguns relatos:

"É a assistência dada ao paciente "incurável" que visa diminuir o sofrimento e que alivie a dor aos pacientes terminais para que ele possa ao seu modo, se preparar para a morte". (T7)

"São todas as medidas instituídas para evitar sofrimento em um paciente internado ou não, no qual não existam perspectivas de tratamento curativo para a doença de base". (M1)

De fato, os CP oferecem não apenas a possibilidade de suspender tratamentos considerados fúteis, mas a realidade tangível de ampliação da assistência oferecida por uma equipe que pode cuidar dos sofrimentos físicos, sintomas de progressão da doença ou das sequelas de tratamentos agressivos que foram necessários no tratamento ou no controle da doença grave e incurável. [12]

A propósito, a questão da futilidade médica também é um grande desafio. Existem razões pelas quais os médicos devem prestar um bom tratamento de suporte de vida, quando indicado, mas não necessariamente para todos os pacientes. Muitos relutam em suspender o tratamento porque temem consequências legais, embora haja declarações e políticas favoráveis, tanto oriundas do Colégio Americano de Emergências Médicas como da Associação Médica Americana.

Em relação às questões ético-legais, o Conselho Federal de Medicina Brasileiro, através da resolução no. 1805/2006, em vigor desde 2010, estabelece a possibilidade de o médico limitar ou suspender procedimentos e tratamentos que prolonguem a vida do doente na fase terminal de enfermidades graves e incuráveis, respeitando a vontade do paciente e de seu representante legal, respaldando, por conseguinte, a decisão médica na escolha pela filosofia de cuidado paliativista [17], corroborando o depoente seguinte:

"São cuidados que visa conforto do paciente em detrimento ao tratamento patológico. A monitorização, digo, a invasão com artefatos é evitada assim como condutas "heroicas", são priorizadas as condutas como o alívio da dor, conforto na terminalidade do paciente". (E12)

Assim, cuidados paliativos exclusivos são definidos como, na fase final de vida, quando a morte se mostra iminente e inevitável, o médico deve oferecer todos os cuidados paliativos disponíveis, sem empreender ações diagnósticas ou terapêuticas inúteis ou obstinadas levando em consideração a vontade expressa do paciente ou seu representante [13].

Nas falas a seguir, apesar dos participantes não trazerem em seus discursos o conceito atual de cuidados paliativos exclusivos, ditam:

"Quando não se investe no paciente de modo a prolongar o sofrimento, não realiza procedimento e exames desnecessários". (T16)

"Apenas analgesia e avaliação dos marcadores de sofrimento. Sem IOT, sem acesso/sonda... sem exames". (F3).

O tratamento fútil, advindo da internação na UTI, proporcionou severa violação à integridade psicofísica do paciente terminal, impossibilitando-o de exercer o direito a uma existência digna. Tal fato logrou vitimar o doente terminal, cujos procedimentos médicos 
invasivos, desvinculados de uma finalidade curativa, proporcionaram-lhe apenas o prolongamento de sua morte e o isolamento daqueles que realmente o amavam [15].

A obstinação terapêutica, ou medicina fútil e inútil ou ainda distanásia é a durame da problemática, que transforma o final da existência do paciente, tornando-os prisioneiros de uma aparelhagem técnica que além de prolongar a vida do ser humano de forma artificial, transforma esses momentos em verdadeiras torturas de dores e sofrimentos. É através desta premissa que os cuidados críticos e intensivos trazem tanto para os pacientes e seus familiares à esperança de uma recuperação "milagrosa", difícil, porém possível, somados ao medo e angústia profunda de perder a própria vida ou há de alguém muito querido, estão inacreditavelmente juntos!

Conclusão

A maioria dos profissionais de saúde que participaram desta pesquisa reconhece a necessidade de qualidade de vida e conforto diante do paciente que tem uma doença que ameace a vida. No entanto, desconhecem os conceitos preconizados sobre cuidados paliativos, e algumas premissas, como quando se deve iniciar, as fases que compõe, além da falta de entendimento sobre o significado dos cuidados paliativos exclusivos.

O trabalho profissional com pacientes com doença que ameace a vida exige formação especial, incluindo capacitação e atualização contínua sobre o assunto, pois é por meio da convivência com esse tema que os profissionais se tornarão cada vez mais habilitados e confiantes na assistência prestada aos pacientes sob seus cuidados. Desta forma, é de suma importância que o tema morte seja tratado de maneira corriqueira, haja vista que com a convivência, a observação, as intervenções e as constantes atualizações a equipe se tornará cada vez mais confiante e apta na realização de sua prática.

Some-se a isto, o que se percebe atualmente nas UTIs é que estas se configuram como espaço de gerenciamento técnico de vidas e mortes. Dessa forma, a perspectiva desafiadora é justamente recuperar o seu papel original, isto é, sua razão de existir, que consiste em aplicar toda a ciência da saúde conhecida em prol da melhora da saúde a da pessoa que está internada concomitante aos cuidados paliativos.

A verdade é que os profissionais de saúde não têm como afastar a morte indefinidamente. A morte chega para todos, esta é a única certeza da vida. Quando a terapia medicamentosa não consegue mais atingir os objetivos de preservar a saúde ou aliviar o sofrimento, tratar para curar torna-se uma futilidade ou um peso e, mais do que prolongar a vida, prolonga-se a agonia. Surge então o imperativo ético de parar o que é inútil e fútil, intensificando os esforços no sentido de proporcionar mais que quantidade, qualidade de vida diante do morrer.

1. Arantes ACQ. A morte é um dia que vale a pena viver. Rio de Janeiro: Casa da Palavra; 2016.

2. Silva RS, Pereira Á, Mussi FC. Conforto para uma boa morte: perspectiva de uma equipe de enfermagem intensivista. Esc Anna Nery 2015;19(1):40-6.

3. Costa ÁP, Poles K, Silva AE. Formação em cuidados paliativos: experiência de alunos de medicina e enfermagem. Interface-Comunicação, Saúde e Educação. 2016;20(59):1041-52. https://doi.org/10.1590/1807-57622015.0774

4. Britto SMC, Ramos RS, dos Santos EI, Veloso OS, da Silva AM, Mariz RGA. Representação social dos enfermeiros sobre cuidados paliativos. Revista Cuidarte 2014; 6(2):1062-9. https://doi.org/10.15649/cuidarte.v6i2.170

5. Coelho MEM, Ferreira AC. Cuidados paliativos: narrativas do sofrimento na escuta do outro. Revista Bioética 2015; 23(2):340-6. https://doi.org/10.1590/198380422015232073

6. Costa JBF, Assunção TAO, Salles HSA. Cuidados paliativos em oncologia pediátrica. Enferm Bras 2017;16(3):177-81. https://doi.org/10.33233/eb.v16i3.1152

7. Silva AF et al. Cuidados paliativos em oncologia pediátrica: percepções, saberes e práticas. Rev Gaúcha Enferm 2015;36(2):56-62.

8. Silva FS, Souza DM, Pedreira LC, dos Santos MR, Faustino TN. Concepções da equipe multiprofissional sobre a implementação dos cuidados paliativos na unidade de 
terapia intensiva. Ciência \& Saúde Coletiva 2013;18(9):2597-604.

https://doi.org/10.1590/s1413-81232013000900014

9. Pessini L. Vida e morte na UTI: a ética no fio da navalha. Rev Bioética 2016;24(1):5463. https://doi.org/10.1590/1983-80422016241106

10. Marconi MA, Lakatos EM. Técnicas de pesquisa. 9 ed. São Paulo: Atlas; 2017.

11. Bardin L. Análise do conteúdo: edição revista e ampliada. São Paulo: Edições 70; 2011.

12. Moritz RD. Cuidados Paliativos nas Unidades de Terapia Intensiva. São Paulo: Atheneu; 2013.

13. Santana JCB dos Santos AC, da Silva BR, Oliveira DCA, Caminha EM, Peres FS, Andrade CCD, Viana MBO. Docentes de enfermagem e terminalidade em condições dignas. Rev Bioética 2013;21(2):298-307. https://doi.org/10.1590/s198380422013000200013

14. Cardoso DH Muniz RM, Schwartz E, Arrieira ICO. Cuidados paliativos na assistência hospitalar: a vivência de uma equipe multiprofissional. Texto Contexto-Enferm 2013;22(4). https://doi.org/10.1590/s0104-07072013000400032

15. Azevedo LCP. Medicina Intensiva: abordagem prática. 2 ed. São Paulo: Manole; 2015.

16. Picanço CM, Sadigursky D. Concepções de enfermeiras sobre o prolongamento artificial da vida. Rev Enferm UERJ 2014;22(5): 668-73.

https://doi.org/10.12957/reueri.2014.15527

17. Brasil. Ministério da Saúde. Resolução CFM no 1805 de 28 de novembro de 2006. Diário oficial da união 2006; 28 nov.

18. Silveira MH, Ciampone MHT, Gutierrrez BAO. Percepção da equipe multiprofissional sobre cuidados paliativos. Rev Bras Geriatr Gerontol 2014;17(1). https://doi.org/10.1590/s1809-98232014000100002 